\title{
Polarization Micro-Optics: circular polarization from a Fresnel Rhomb 3D printed on an optical fiber
}

\author{
Andrea Bertoncini, Carlo Liberale
}

\begin{abstract}
High resolution fabrication of three dimensional polymeric structures with Direct Laser Writing (DLW) holds a unique potential for the miniaturization of bulk optical elements. This has been so far mainly demonstrated for the fabrication of reflective, refractive and phase-mask micro-optics. Here we show the use of DLW for the fabrication of a miniaturized phase retarder element, a Fresnel Rhomb, which acts as a broadband quarter-wave plate. We show the integration of the Fresnel Rhomb onto a polarization-maintaining optical fiber, converting linearly polarized light from the fiber into circularly polarized light over a very broad spectral range.
\end{abstract}

Index Terms-: Prisms; Micro-optics; Three-dimensional lithography; Fiber optics; Fibers, polarization-maintaining.

\section{INTRODUCTION}

$\mathrm{T}$ HERE has been a consistent effort to find methods to fabricate and integrate miniaturized optical elements which can parallel the function of bulk optical elements used in large scale optical set-ups and systems. This quest is spurred by the need to create compact and integrated optical systems for a number of devices, such as components for optical telecommunications, Point of Care devices and remote optical probes. This has led to the emergence of Direct Laser Writing (DLW) based on multiphoton absorption [1,2] as an effective method to create micro-optics based on polymer materials. This method is essentially a high-resolution 3D printing technique with a sub-micron resolution [3], which is based on the 3D localized polymerization of a suitable resin through multiphoton absorption of a tightly focused laser beam. Thanks to its unprecedented combination of small scale, arbitrary 3D shapes and simplicity of the fabrication process, DLW based on multiphoton absorption has found application in very different areas such as photonics [4], micro-mechanics [5], microfluidics [6], electronics [7], and biology [8].

In particular, with this technique, different reflective, refractive and diffractive micro-optic elements have been fabricated with satisfactory optical quality for visible and nearinfrared wavelengths, including prisms [9], lenses [10, 11], parabolic mirrors [12], gratings [13] and phase-masks [14]. A

This work was supported by the King Abdullah University of Science and Technology (KAUST) under baseline funding BAS/1/1064-01-01

A. B. and C.L are with the Biological and Environmental Science and Engineering (BESE) and Computer, Electrical and Mathematical Sciences and Engineering (CEMSE) Divisions, King Abdullah University of Science and Technology (KAUST), Thuwal 23955-6900, Saudi Arabia

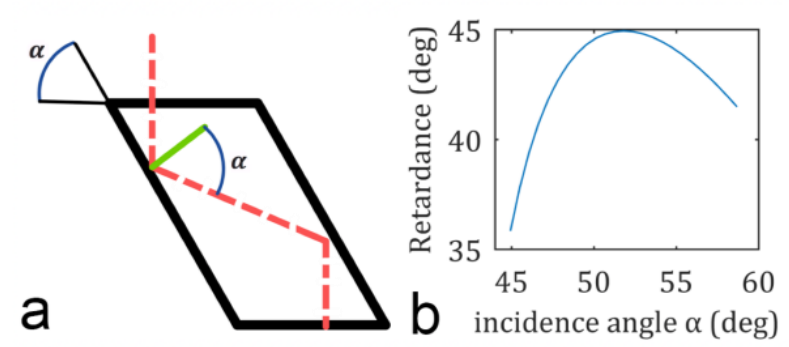

Fig. 1. (a) Sketch of the Fresnel Rhomb with a ray (dashed line) incident at normal angle to the small side of the parallelogram (b) Phase shift difference between $\mathrm{s}$ and $\mathrm{p}$ polarization components upon single reflection with respect to incidence angle $\alpha$, as calculated for the Fresnel Rhomb presented here.

noticeable feature of DLW is the ability to fabricate directly on the substrate or device where the optical element should be placed, often making straightforward the integration process. This has been used, for example, to easily fabricate optical elements directly on the tip of optical fibers, such as Spiral Phase Plates for the generation of Orbital Angular Momentum [14], lenses and phase plates for single mode fiber beam shaping [15], parabolic mirrors to increase the Numerical Aperture in multimode fiber based endoscopy [16], and reflective prisms on multicore fibers for single cell trapping and spectroscopy [17].

However, no miniaturized phase retarder element has been so far proposed based on DLW 3D printing.

Here we show the use of DLW for the fabrication of a miniaturized phase retarder element, the Fresnel Rhomb [18]. The Fresnel Rhomb is a rhombohedral prism in which light experiences two total internal reflections at a precisely designed incidence angle $(\alpha)$ (Fig. 1(a)) so that the total phase shift difference between $s$ and $p$ components of polarized light at the output of the prism is $90^{\circ}$ [18]. The Fresnel rhomb thus acts as a quarter-wave plate with a phase shift which is not based on material birefringence and therefore features a very broad spectral bandwidth. Alternatively, a broadband half-wave plate can also be created by using a stack of two Fresnel prisms. With reference to Fig 1a, when a ray enters the rhomb perpendicular to one of the small sides of the parallelogram, then the incidence

*Corresponding author: carlo.liberale@kaust.edu.sa Copyright (c) 2018 IEEE. Personal use of this material is permitted. However, permission to use this material for any other purposes must be obtained from the IEEE by sending a request to pubspermissions@ieee.org. 
angle at the two oblique-angled sides is equal to the acute angle of the parallelogram $(\alpha)$.

As an exemplary application of this miniaturized Fresnel Rhomb, we have fabricated a compact (320 $\mu \mathrm{m}$ long) on-fiber quarter wave plate on the output face of a PolarizationMaintaining (PM) optical fiber [19], by aligning at 45 degrees the rhomb axes with respect to the PM fiber axes. By aligning the input polarization along the slow or fast axis of the PM fiber, the on-fiber Fresnel Rhomb can generate left or right circular polarization over a large spectral bandwidth. In this article we describe thoroughly the design and fabrication process of the device presented in [19]. Moreover, we demonstrate the very broad operational bandwidth of the device by performing a spectral measurement of the Stokes parameters.

The broad bandwidth featured by this structure (> $300 \mathrm{~nm}$ ) could be exploited to create an ultra-compact fiber probe for remote excitation in circular dichroism based spectroscopies, such as circular dichroism (in the UV-VIS band) and vibrational circular dichroism (NIR-IR band), as well as for Raman Optical Activity spectroscopy. We note that the wide bandwidth requirement for these applications would rule out fiber-based quarter waveplates (which are PM fibers cut at a length corresponding to a quarter of their beat length and spliced to another PM fiber, with a 45 degrees angle between their axes [20]) because of their limited spectral bandwidth (on the order of $50 \mathrm{~nm}$ at $1000 \mathrm{~nm}$ wavelength). Of note, an approach is reported to obtain a broadband fiber quarter-wave plate by using a variably spun PM fiber with a slowly varying spin rate [21]. However, these structures are not reciprocal, and a minimum length of a few centimeters is still required.

\section{MATERIALS AND MethodS}

To design the Fresnel Rhomb, we have started from the equations that describe the phase shift difference $\Delta$ between the $\mathrm{p}$ and s polarization components upon total internal reflection [21]:

$$
\begin{aligned}
& \delta_{s}=\frac{\sqrt{n^{2} \sin ^{2} \alpha-1}}{n \cos \alpha} \\
& \delta_{p}=2 \tan ^{-1} \frac{n \sqrt{n^{2} \sin ^{2} \alpha-1}}{\cos \alpha} \\
& \Delta=\delta_{p}-\delta_{s}
\end{aligned}
$$

where $\delta_{s}$ and $\delta_{p}$ are the phase shifts for the $s$ and $p$ polarization components, respectively, $n$ is the refractive index of the Fresnel Rhomb, and $\alpha$ is the incidence angle and the acute angle of the parallelogram.

By using the above equations and the refractive index of the crosslinked photopolymer (IP-S, Nanoscribe $\mathrm{GmbH}$ ), as retrieved from [22], an optimum angle of $\alpha=51.82 \mathrm{deg}$, has been determined, at which the phase shift difference between $p$ and $s$ polarization components is $\pi / 8$ at the design wavelength of 935 nm (Fig. 1(b)).

As the phase shift difference $\Delta$ is significantly dependent on the incidence angle $\alpha$, without collimation of the divergent beam output from the fiber the collimated beam exiting the
Fresnel Rhomb would show a nonuniform polarization state across its transverse dimension. To solve this problem, we let the beam output for the fiber to freely expand until it reaches a beam radius of $13 \mu \mathrm{m}$ after $150 \mu \mathrm{m}$ of propagation in the

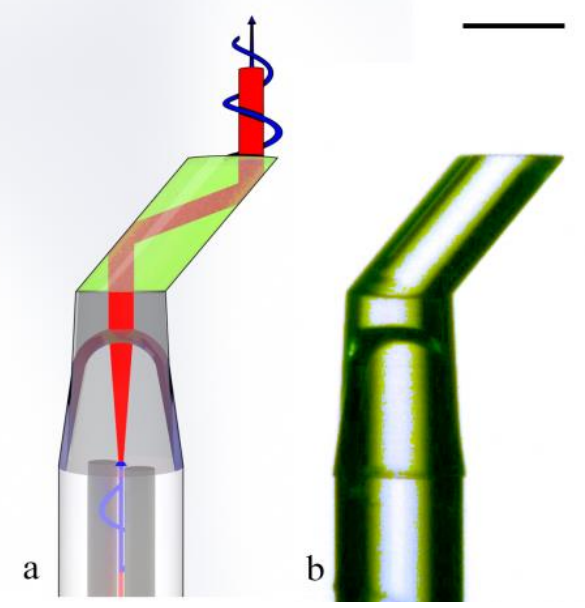

Fig. 2. (a) Designed structure composed of two main parts: a collimating microlens and a Fresnel Rhomb, oriented at $45 \mathrm{deg}$ with respect to the optical axis of the PM fiber. (b) Optical image of the fabricated structure. Scale bar is $100 \mu \mathrm{m}$.

polymerized resin, and then we collimate it by using a properly designed spherical micro-lens (Fig. 2(a)). We calculated a radius of curvature of $50 \mu \mathrm{m}$ for the collimating lens by using the ABCD formalism and the refractive index of crosslinked photopolymer. It is worth noticing here that $3 \mathrm{D}$ printing by DLW uniquely allows this flexibility of combining two geometrically different optical elements such as the micro-lens and a micro Fresnel Rhomb on the same device and during the same fabrication process.

The beam collimating segment (blue in Fig. 2(a)) of the designed micro-structure has the same diameter of the optical fiber $(125 \mu \mathrm{m})$ and is $150 \mu \mathrm{m}$ tall. The input and output faces of the Fresnel Rhomb have a dimension of 90 by $125 \mu \mathrm{m}$. The gap between the beam collimating segment and the Fresnel Rhomb is $40 \mu \mathrm{m}$.

Fabrication of the structures is performed by using a a commercial DLW 3D printer based on two-photon photopolymerization (Photonic Professional GT, Nanoscribe $\mathrm{GmbH}$ ) with an immersion objective (Zeiss, LCI PlanNEOFLUAR 25x/0.8 Imm Corr) and the proprietary IP-S resist (Nanoscribe $\mathrm{GmbH}$ ) in dip-in configuration [23]. This configuration provides sub-micron resolution [6, 24], high optical quality [10] and high mechanical stability [24].

A quarter-wave plate structure has been eventually fabricated on top of a PM Panda-style optical fiber with $\mathrm{NA}=0.12$ (PM780-HP, Thorlabs) (Fig. 2(b)) .

After terminating the PM fiber with a connector with alignment key (B30126C3, Thorlabs), we have mounted it on to a custom fiber holder that includes a terminated fiber adapter (SM1FC, Thorlabs). The IP-S resist is then directly drop cast on the optical fiber and, finally, the objective is dipped into the resist.

Fine alignment of the optic axis of the PM fiber with respect to the $3 \mathrm{D}$ printer coordinate system is achieved thanks to the 


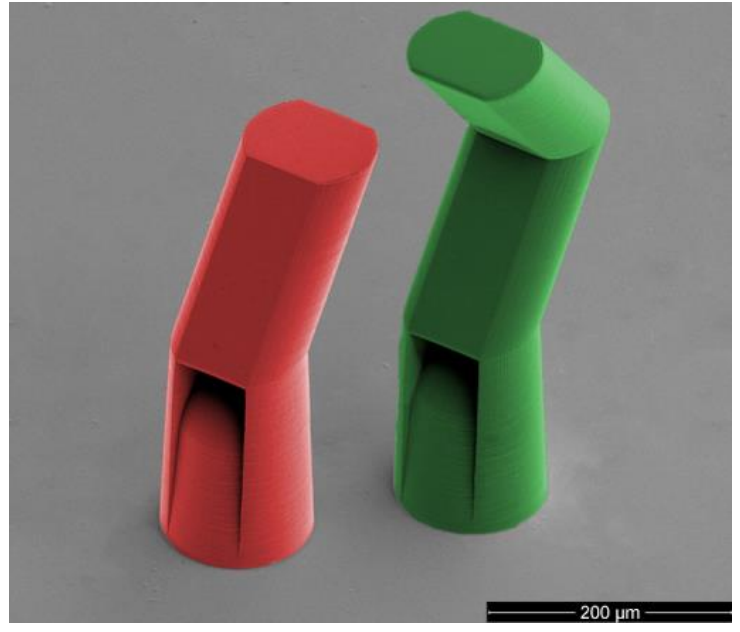

Fig. 3. Scanning electron microscope image of a quarter-waveplate structure (with only one Fresnel Rhomb, left) and of a half-waveplate structure (with two stacked Fresnel Rhombs, right) printed on a glass substrate.

built-in camera with reflection illumination, which allows a clear observation of the fiber stress rods that mark the fast axis of the PM fiber.

\section{RESULTS AND DISCUSSION}

To assess the quality of the fabricated elements and to show the potential to fabricate also half-wave plate structures, we have printed of a quarter-wave plate structure (with only one Fresnel Rhomb) and a half-wave plate (hence with two stacked Fresnel Rhombs) on a glass substrate and performed scanning electron microscopy (Fig. 3). It can be clearly appreciated the precision of fabrication and the high optical quality of the surfaces.

To characterize the optical performance of our device, we have measured the space-average of the normalized Stokes parameters $S=\{S 0, S 1, S 2, S 3\}$ for the output beam [25]. For every spatial point on the cross-section of the beam, the four normalized Stokes parameters fully describe the polarization ellipse. A perfectly circular right-hand or left-hand polarization state will result in an S3 parameter equal to +1 or -1 respectively. Therefore, when measuring the space-average of the Stokes parameters, we expect to find an absolute value of the space-averaged S3 parameter equal to 1 only in the case of spatially uniform and purely circular polarization.

The measurement of the space-averaged Stokes parameters has been performed with a setup that derives from the classical method involving the use of a quarter-wave plate plus a polarizer, as described in [25], and a bucket detector, which intrinsically performs a spatial integration of the local beam intensities. In this method, a set of four different beam powers are measured at four different conditions of the polarizer and the wave plate and then are used to retrieve the space-average of the four Stokes parameters by using simple formulas [25].

A tunable laser (Charmeleon Discovery, Coherent) is coupled to the PM fiber with a 10x objective (Mplan N Olympus, $0.25 \mathrm{NA}$ ) and with the beam polarization aligned to one of the fiber's principal axes. The output of the on-fiber Fresnel Rhomb is collected by a 10x objective (CFI Plan Achro

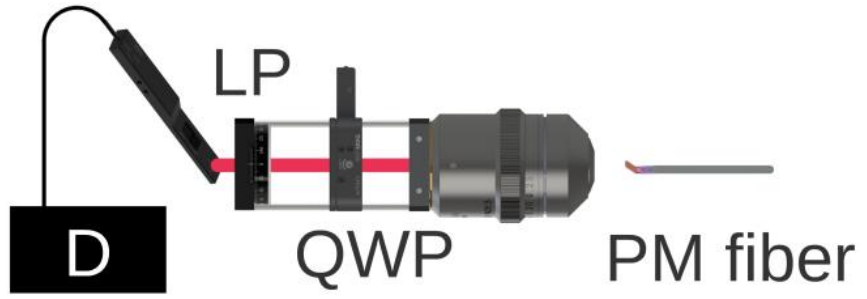

Fig. 4. Setup used to measure the Stokes parameters. QWP: quarter-wave plate; LP: linear polarizer; D: detector, PM fiber: Polarization maintaining fiber.

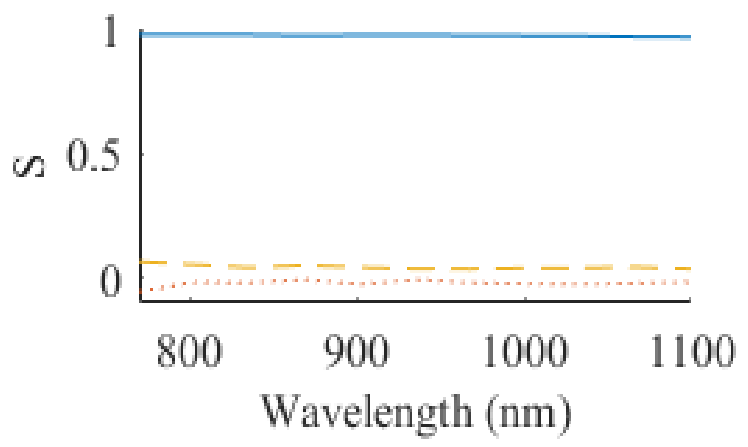

Fig. 5. Normalized Stokes parameters measured between $770 \mathrm{~nm}$ and 1100 nm. Continuous line S3, dotted line S2, dashed line S1.

Nikon, 0.25 NA, recommended by the manufacturer for polarization sensitive applications) and passed through an achromatic quarter waveplate (AQWP05M-980, Thorlabs) and a polarizer (LPNIRE100-B, Thorlabs) and finally measured with a power meter (Fig. 4). The spectral range of the characterization has been limited by the specified spectral bandwidth of the PM fiber, which is 770-1100 nm.

Four optical powers (P1, P2, P3, P4) have been measured in different conditions [21]. The first three powers are measured with, the quarter-wave plate out of the optical path, and with the polarizer rotated at $0^{\circ}, 90^{\circ}$, and $45^{\circ}$, respectively. Then, the $\mathrm{P} 4$ power is measured with the polarizer at $45^{\circ}$, and by inserting the quarter-waveplate aligned to the horizontal axis.

The resulting Stokes parameters are then calculated as:

$$
\begin{aligned}
& S O=P 1+P 2 \\
& S 1=P 1-P 2 \\
& S 2=2 * P 3-S O \\
& S 3=2 * P 4-S O
\end{aligned}
$$

The measured spatially averaged S3 as a function of the wavelength is reported in Fig. 5, and clearly indicates an output beam with a spatially homogenous and nearly pure circular polarization. Of note, the phase retardance for the used achromatic quarter waveplate, as specified by the manufacturer over the measured spectral bandwidth, is having only a negligible effect on this measurement.

To prove the necessity of beam collimation to obtain a spatially uniform circular polarization, we have also printed the Fresnel Rhomb without a collimating micro-lens at the output of the fiber. The resulting spatially averaged normalized Stokes parameters measured at $1064 \mathrm{~nm}$ are $\{\mathrm{S} 0=1, \mathrm{~S} 1=-0.07, \mathrm{~S} 2=-$ $0.018, \mathrm{~S} 3=-0.32\}$, which indicates that the beam is far from 
having a spatially uniform and purely circular polarization state.

\section{CONCLUSION}

In conclusion, we have shown the fabrication of a miniaturized and broadband phase retarder by $3 \mathrm{D}$ printing of a Fresnel Rhomb with DLW. We have also shown an exemplary device which includes this structure by creating a fiber probe which delivers a beam with nearly pure circular polarization over a very large bandwidth (>300 nm). Of note, by considering the dispersion coefficients for the photoresist, as provided in [22], we can theoretically predict an larger operational spectral range for the miniaturized Fresnel Rhomb, with a lower wavelength limit of about $550 \mathrm{~nm}$.

We anticipate that the miniaturized Fresnel Rhomb could find application in circular dichroism and Raman Optical Activity spectroscopies but could also be used as a phase retarder for other integrated applications, where control of the polarization on small scales is needed, e.g. for pixel-level circular polarizers in image sensors or control of circular polarization generation in VCSEL arrays.

Finally, we point out that, while the here presented fiber probe delivers a free-space circular polarized beam, the flexibility of DLW 3D printing would allow fabricating a second focusing lens after the Fresnel Rhomb to couple the light into another optical fiber which can be mechanically spliced as shown in [26].

\section{REFERENCES}

[1] M. Malinauskas, M. Farsari, A. Piskarskas, and S. Juodkazis, "Ultrafast laser nanostructuring of photopolymers: A decade of advances", Physics Reports-Review Section of Physics Letters, vol. 533, no. 1, pp. 1-31, Dec 12013.

[2] H. B. Sun and S. Kawata, "Two-photon photopolymerization and 3D lithographic microfabrication" Nmr - 3d Analysis Photopolymerization, vol. 170, pp. 169-273, 2004.

[3] Z. S. Gan, Y. Y. Cao, R. A. Evans, and M. Gu, "Three-dimensional deep sub-diffraction optical beam lithography with $9 \mathrm{~nm}$ feature size" Nature Communications, vol. 4, Jun 2013.

[4] N. Lindenmann et al., "Photonic wire bonding: a novel concept for chip-scale interconnects", Optics Express, vol. 20, no. 16, pp. 17667-17677, Jul 302012.

[5] T.-T. Chung, C.-L. Tseng, C.-P. Hung, C.-L. Lin, and P. L. Baldeck, "Design and two-photon polymerization of complex functional micro-objects for lab-on-a-chip: Rotating micro-valves" Journal of Neuroscience and Neuroengineering, vol. 2, no. 1, pp. 48-52, 2013.

[6] G. Nelson et al., "Three-dimensional-printed gas dynamic virtual nozzles for x-ray laser sample delivery" Optics Express, vol. 24, no. 11, pp. 1515-1530, May 302016.

[7] K. Kurselis, R. Kiyan, V. N. Bagratashvili, V. K. Popov, and B. N. Chichkov, "3D fabrication of all-polymer conductive microstructures by two photon polymerization" Optics Express, vol. 21, no. 25, pp. 31029-31035, Dec 162013.

[8] F. Klein et al., "Two-Component Polymer Scaffolds for Controlled Three-Dimensional Cell Culture", Advanced Materials, vol. 23, no. 11, pp. 1341-1345, Mar 182011.

[9] M. Malinauskas et al., "Laser fabrication of various polymer microoptical components", European Physical Journal-Applied Physics, vol. 58, no. 2, May 2012.

[10] T. Gissibl, S. Thiele, A. Herkommer, and H. Giessen, "Twophoton direct laser writing of ultracompact multi-lens objectives" Nature Photonics, vol. 10, no. 8, pp. 554, Aug 2016.

[11] P. I. Dietrich et al., "In situ 3D nanoprinting of free-form coupling elements for hybrid photonic integration" Nature Photonics, vol. 12, no. 4, pp. 241-247, 2018/04/01 2018.
[12] J. H. Atwater et al., "Microphotonic parabolic light directors fabricated by two-photon lithography", Applied Physics Letters, vol. 99, no. 15, Oct 102011.

[13] H. C. Guo et al., "Two-photon polymerization of gratings by interference of a femtosecond laser pulse" , Chemical Physics Letters, vol. 374, no. 3-4, pp. 381-384, Jun 112003.

[14] E. Brasselet, M. Malinauskas, A. Žukauskas, and S. Juodkazis, "Photopolymerized microscopic vortex beam generators: precise delivery of optical orbital angular momentum" Applied Physics Letters, vol. 97, no. 21, p. 211108, 2010.

[15] C. Liberale et al., "Micro-Optics Fabrication on Top of Optical Fibers Using Two-Photon Lithography", Ieee Photonics Technology Letters, Article vol. 22, no. 7, pp. 474-476, APR 1 20102010.

[16] S. Bianchi, V. Rajamanickam, L. Ferrara, E. Di Fabrizio, C. Liberale, and R. Di Leonardo, "Focusing and imaging with increased numerical apertures through multimode fibers with micro-fabricated optics", Optics Letters, Article vol. 38, no. 23, pp. 4935-4938, DEC 120132013.

[17] C. Liberale et al., "Integrated microfluidic device for single-cell trapping and spectroscopy", Scientific Reports, Article vol. 3, FEB 132013 2013, Art. no. ARTN 1258.

[18] A. J. Fresnel, "Memoire sur les modifications que la reflexion imprime ala lumiere polarisse" $F O$, vol. 1, p. 441, 1817.

[19] A. Bertoncini and C. Liberale, "3D printed Polarization MicroOptics: Fresnel Rhomb printed on an optical fiber" in Integrated Photonics Research, Silicon and Nanophotonics, 2018, p. ITh4B. 6: Optical Society of America.

[20] S. X. Short, A. A. Tselikov, J. U. de Arruda, and J. N. Blake, "Imperfect quarter-waveplate compensation in Sagnac interferometer-type current sensors", Journal of Lightwave Technology, vol. 16, no. 7, pp. 1212-1219, Jul 1998.

[21] H. C. Huang, "Fiber-optic analogs of bulk-optic wave plates", Applied Optics, vol. 36, no. 18, pp. 4241-4258, Jun 201997. T. Gissibl, S. Wagner, J. Sykora, M. Schmid, and H. Giessen, "Refractive index measurements of photo-resists for threedimensional direct laser writing" Optical Materials Express, vol. 7, no. 7, pp. 2293-2298, 2017.

[23] T. Buckmann et al., "Tailored 3D Mechanical Metamaterials Made by Dip-in Direct-Laser-Writing Optical Lithography", Advanced Materials, vol. 24, no. 20, pp. 2710-2714, May 222012.

[24] Y. Liu, O. Stein, J. H. Campbell, L. J. Jiang, N. Petta, and Y. F. $\mathrm{Lu}$, "Three-dimensional printing and deformation behavior of lowdensity target structures by two-photon polymerization", Nanoengineering: Fabrication, Properties, Optics, and Devices Xiv, vol. 10354, 2017.

[25] D. H. Goldstein, Polarized Light, revised and expanded. CRC press, 2003.

S. Kedenburg, T. Gissibl, T. Steinle, A. Steinmann, and H. Giessen, "Towards integration of a liquid-filled fiber capillary for supercontinuum generation in the 1.2-2.4 mu m range", Optics Express, vol. 23, no. 7, pp. 8281-8289, Apr 62015. 